\title{
IMPLEMENTATION FO AUSTRALIA'S 1ST WEBCAM IN A NICU
}

\author{
Z. Kecskes ${ }^{1,2}$, B. Connors ${ }^{3}$ \\ ${ }^{I}$ Dept of Neonatology, Canberra Hospital, ${ }^{2}$ Medical School, Australian National University, Canberra, \\ ${ }^{3}$ Dept of Neonatology, Canberra Hospital, Garran, ACT, Australia
}

Background: The implications for families of a baby cared for in a neonatal intensive care unit include physical and emotional separation and alienation from their baby, which strains the early parent-infant bonding process. This may lead to poor parental empathy for the child thereby increasing the risk of child abuse.The aim of NICUcam in the Centre for Newborn Care was to help parents to get to know their babies, to inform parents of their baby's status, to build confidence in their ability to care for them once they leave the hospital and to improve early parent-infant bonding.

Method: The project was funded by ACT Government. The project development included legal advice, close collaboration with ANU and ACT Health library for website design and management, as well as consumer input. Guideline development, education, emergency procedures and risk assessment were put into place for the project. The project consisted of two phases and was backed by strong corporate commitment.

Results: The pilot project commenced December 2009; the project was launched in January 2010. Over a 12 month period, 70 families have been using the webcam. The website has been accessed 30,000 times, the cameras 10,800 times with viewers from 20 countries. Parental access is granted by unique username and password, viewing hours are twice a day and feedback is received via the internet.

Conclusion: This system allows families to view their baby remotely, hopefully improving early bonding and reduction of LOS in the future. 